ENCYCLOPÉDIE Encyclopédie berbère

BERBERE

28-29 | 2008

28-29 | Kirtēsii - Lutte

\title{
Les Laguatan
}

Le problème des migrations des « néo-Berbères »

\section{Y. Modéran}

\section{OpenEdition}

\section{Journals}

Édition électronique

URL : http://journals.openedition.org/encyclopedieberbere/288

DOI : $10.4000 /$ encyclopedieberbere.288

ISSN : 2262-7197

\section{Éditeur}

Peeters Publishers

\section{Édition imprimée}

Date de publication : 1 janvier 2008

Pagination : 4318-4321

ISBN : 2-7449-0707-4

ISSN : $1015-7344$

\section{Référence électronique}

Y. Modéran, « Les Laguatan », Encyclopédie berbère [En ligne], 28-29 | 2008, document L03b, mis en ligne le 01 juin 2013, consulté le 24 septembre 2020. URL : http://journals.openedition.org/ encyclopedieberbere/288; DOI : https://doi.org/10.4000/encyclopedieberbere.288

Ce document a été généré automatiquement le 24 septembre 2020.

(c) Tous droits réservés 


\section{Les Laguatan}

Le problème des migrations des « néo-Berbères »

\section{Y. Modéran}

1 Les recherches de D. J. Mattingly ont, depuis 1983, considérablement éclairci les problèmes posés par l'histoire des Laguatan, et il n'est pas dans mon propos ici de revenir sur l'ensemble du sujet. Le but de cette note contradictoire, fidèle à une tradition inaugurée par G. Camps et P.-A. Février avec la notice "Abizar», est simplement de proposer, pour un certain nombre de points qui restent en discussion, une interprétation différente, pour laquelle on trouvera des développements plus importants dans mon livre Les Maures et l'Afrique romaine (IVe-VII siècle), cité dans la bibliographie de D. J. Mattingly.

2 Trois questions restent principalement objets de débat. La première concerne l'origine des Laguatan. Sans entrer dans les détails, D. J. Mattingly estime qu'ilsvenaient de l'est du Maghreb, des confins de l'Égypte, et qu'ils appartenaient à un groupe de tribus qualifiées de "néo-Berbères", engagées dès le $\mathrm{III}^{\mathrm{e}}$ siècle dans un mouvement de migration. Or, cette interprétation me semble contestable pour au moins deux raisons majeures. Ce terme même de "néo-Berbères", popularisé par G. Camps, désigne d'abord une spécificité tribale qui n'est attestée, en réalité, par aucun texte, en tout cas pour l'Antiquité. Loin d'être le reflet d'un acquis scientifique indiscutable, il n'est que l'expression la plus remarquable d'une thèse plus large sur la bipartition ethnique du monde berbère, attestée seulement au MoyenÂge par Ibn Khaldûn, et que celui-ci exprime selon un schéma généalogique distinguant Botr et Branès. Ce n'est que par un raisonnement à la fois rétroactif et circulaire que l'on fait de ce concept de néoBerbères une explication de l'apparition des Laguatan (Modéran 2001 et 2003).

3 La mention de la guerre des Marmarides de la fin des années 260 comme premier moment de l'émergence historique de ces Laguatan constitue, d'autre part, un choix discutable, car les Marmarides sont connus sous ce nom depuis longtemps aux confins de l'Égypte, et on les y retrouve encore plus tard. Ces gens formaient même probablement aussi une confédération, et J. Desanges (2001) a proposé, avec de bons arguments, d'identifier parmi eux des Gôniôtae, mentionnés par plusieurs documents papyrologiques du $\mathrm{III}^{\mathrm{e}}$ siècle, et connus pour leur activité belliqueuse peu avant la 
guerre de 268-269.Vouloir retrouver les Laguatan ou des ancêtres des Laguatan derrière ces Marmarides est une pure hypothèse, en tout cas pour cette époque. Or, sans eux, plus rien n'appuie vraiment la thèse de l'origine orientale.

4 Si l'on s'en tient aux textes antiques, on constate seulement, en effet, qu'à partir de la fin du III ${ }^{e}$ siècle et surtout au $\mathrm{IV}^{\mathrm{e}}$ siècle apparaissent des tribus appelées Laguatan et Austuriani, qui menacent la Tripolitaine d'abord, puis la Cyrénaïque, en partant de bases situées très au sud ou au sud-est. J'ai proposé de voir en elles deux fractions de l'ancienne confédération des Nasamons, disloquée après les coups que lui portèrent les Romains à la fin du $\mathrm{I}^{\mathrm{er}}$ siècle, et recomposée désormais sous une nouvelle forme, en ayant conservé comme principal foyer l'oasis d'Augila.

5 Un deuxième point qui ne peut que susciter les discussions tient à la prétendue migration vers l'ouest des Laguatan et des Austuriani. On vient de voir qu'ils apparaissent d'abord en Tripolitaine, peut-être à la fin du $\mathrm{III}^{\mathrm{e}}$ siècle, et à coup sûr au milieu du IV ${ }^{e}$ siècle ( $n^{\circ} 2$ et 3 dans la liste de Mattingly). Ils menacent ensuite la Cyrénaïque $\left(\mathrm{n}^{\circ} 4\right)$ au début du $\mathrm{V}^{\mathrm{e}}$ siècle, puis à nouveau la Tripolitaine aux $\mathrm{V}^{\mathrm{e}}$ et $\mathrm{VI}^{\mathrm{e}}$ siècles ( $n^{\circ} 5,7$, et 9), avant de réapparaître, à l'arrivée des Arabes, en Cyrénaïque et en Tripolitaine. Tout montre ainsi qu'ils ne cessèrent, à partir de foyers qui se rapprochèrent peu à peu de la côte, d'exercer une pression sur les cités des deux provinces de l'actuelle Libye, en réalisant une progression orientée non d'est en ouest, mais bien plutôt du sud vers le nord, ou, si l'on veut, du sud vers le nord-est et le nordouest. La seule anomalie pour toute l'Antiquité réside dans les raids répétés menés de 544 à 548 en Byzacène et jusqu'en Proconsulaire, dans la Tunisie actuelle $\left(n^{\circ} 9\right)$. Mais il s'agit là d'événements guerriers exceptionnels, sans précédents, et sans suites. Aucun document n'autorise à supposer qu'une voie de migration était alors ouverte, qui aurait permis un lent glissement des Laguatan vers la Tunisie à l'époque byzantine. Le numéro 8 de la liste de Mattingly (Procope, III, 25) me semble, à cet égard, fort contestable car, outre que, dans ce texte où Procope raconte les premiers accords, en Tunisie du Nord, entre Byzantins et Maures en 533, leur nom n'apparaît pas, on remarque aussi que l'historien grec mentionne plusieurs provinces comme terre d'origine de ces Maures désireux de négocier, mais précisément pas la Tripolitaine! Je ne vois pas, de même, ce qui justifie l'assimilation de Cabaon $\left(n^{\circ} 6\right)$ à un Laguatan : c'est une hypothèse, parmi bien d'autres possibles.

6 En réalité, ici aussi, notre vision est faussée par les textes arabes médiévaux, qui, surtout à partir d'Al-Bakrî, citent effectivement des tribus Louata un peu partout au Maghreb. Mais ces textes sont très tardifs, et rien n'autorise, comme Jacques Berque l'a montré dès 1954, à identifier ces multiples groupes Louata comme autant « d'alluvions émiettées ", du III ${ }^{e}$ au XIV ${ }^{\mathrm{e}}$ siècle, de la marche d'un grand peuple vers l'ouest (Berque 1954 et 1978) : ils appartiennent à un autre monde, qui possédait ses propres systèmes classificatoires.

7 Le troisième point en débat est relatif à la nature et à l'étendue de la confédération Laguatan. D. J. Mattingly estime que presque toutes les tribus citées par Corippus dans son catalogue des tribus insurgées en 546 en faisaient partie ( $c f$. la référence à Johannide, II, 7-148, pour illustrer "the complex tribal hierarchy of the confederation »), en soulignant cependant, en fin d'article, le caractère extrêmement souple de cette structure. Tout le problème tient ici à la manière dont Corippus use de l'ethnonyme Laguatan (ou Ilaguas). On peut considérer, comme Mattingly, que le poète l'emploie toujours avec un sens ethnographique précis, en désignant une 
confédération. Mais dans ce cas, il faut suivre cette logique jusqu'au bout et dire aussi, en fonction de Johannide, I, 466-467 (Antalas, Laguatan gentis acerbae ductor magnanimus), comme Courtois l'avait vu (Les Vandales et l'Afrique, p. 345), qu'Antalas et ses hommes étaient des Laguatan. Or c'est évidemment absurde, Mattingly le reconnaît, et Procope, notamment, le dément absolument (Guerre vandale, II, 21, 16-18, et II, 28, 47). La seule solution dès lors est, me semble-t-il, d'admettre, comme j'ai essayé de démontrer en accord avec Vincent Zarini (1997), que le poète use parfois du nom Laguatan dans un sens très général, pour qualifier les Maures insurgés au sens large, en choisissant ce nom, celui des adversaires les plus puissants et les plus lointains des Byzantins, pour mieux souligner leur barbarie. Cette ambivalence de certains ethnonymes est, de manière générale, une des difficultés majeures de la Johannide, qui est d'abord une œuvre poétique. Mais une fois perçue, elle permet de restituer sa cohérence historique au poème, et explique surtout pourquoi, dans le fameux catalogue du chant II (dont la structure interne, complexe, est à mon sens très révélatrice), les Laguatan et les Austur (iani), eux-mêmes déjà grossis depuis plus ou moins longtemps de populations de l'arrière-pays des cités de Tripolitaine, sont bien distingués des tribus de l'extrême ouest de la province, et a fortiori des groupes qui suivaient Antalas et Iaudas, le chef de l'Aurès.

$8 \mathrm{Au}$ fond, l'écart entre les deux thèses en présence sur cette question n'est cependant pas si important: il tient simplement à la différence qu'on peut établir entre une alliance et un lien confédéral. D. J. Mattingly est très attaché à cette dernière notion. Empruntée à l'ethnographie moderne, elle n'est cependant guère étayée, dans sa conception large, par les textes, et notre collègue en est bien conscient, en écrivant à la fin de son étude : « it was only in quite exceptionnal circumstances that the principle of tribal confederation would have operated at its fullest extent». Si on la restreint, comme la Johannide nous invite à la faire, et que l'on reconnaît surtout dans les événements de 544-548, hors de la Tripolitaine proprement dite, des alliances, un accord entre nos deux interprétations peut aisément être trouvé.

\section{BIBLIOGRAPHIE}

BERQUE J., « Qu'est-ce qu'une tribu nord-africaine ? » Hommage à Lucien Febvre, Paris, 1954, p. 261-271.

BERQUE J., L'intérieur du Maghreb, XV'-XIXe siècle, Paris, 1978.

DESANGES J., « De la Marmarique à la Maurétanie. Nouvelles données en matière de mouvements de population », Antiquités africaines, 37, 2001, p. 81-91.

MODÉRANY., « Mythe et histoire aux derniers temps de l'Afrique antique : à propos d'un texte d'Ibn Khaldûn ", Revue historique, t. CCCIII/2, 2001, p. 315-341.

ZARINI V., Berbères ou barbares? Recherches sur le livre second de la Johannide de Corippe, Nancy, 1997. 
INDEX

Mots-clés : Antiquité, Histoire, Libye, Moyen Âge, Tribu(s) 